\title{
Rhodococcus opacus strain PD630 as a new source of high-value single-cell oil ? Isolation and characterization of triacylglycerols and other storage lipids
}

\author{
Marc Wältermann, ${ }^{1}$ Heinrich Luftmann, ${ }^{2}$ Dirk Baumeister, ${ }^{1}$ \\ Rainer Kalscheuer ${ }^{1}$ and Alexander Steinbüchel ${ }^{1}$
}

Institut für Mikrobiologie der Westfälischen

Wilhelms Universität ${ }^{1}$ and Institut für Organische Chemie der Westfälischen Wilhelms Universität², Münster, Germany

\author{
Author for correspondence: Alexander Steinbüchel. Tel: +49251833 9821. Fax: +492518338388. \\ e-mail: steinbu@uni-muenster.de
}

The triacylglycerol (TAG)-accumulating, hydrocarbon-degrading bacterium Rhodococcus opacus strain PD630 and chemically induced storage-deficient mutants derived from this strain were investigated for their capability to accumulate storage lipids in the cytoplasm during cultivation under nitrogenlimiting conditions. Acylglycerols were analysed by matrix-associated laser desorption ionization-time of flight mass spectrometry (MALDI-TOF) and by reversed-phase HPLC. Fatty acids comprising 13-19 carbon atoms in various acylglycerols constituted up to $76 \%$ of the cellular dry weight in gluconategrown cells, with a significant proportion of odd-numbered fatty acids. Hydrolysis using pancreatic lipase and deacylation with ethyl magnesium bromide were employed to identify the stereospecific distribution of fatty acids at the glycerol. This analysis showed that the fatty acids were not randomly distributed between the three positions of the glycerol backbone. In comparison with common plant fats, where the longer and higher unsaturated fatty acids are predominantly found at position 2, R. opacus PD630 accumulated only the shorter and saturated fatty acids in this position. More than 100 mutants accumulating TAG at a significantly lower rate were obtained by chemical mutagenesis and identified by staining with Sudan Black $B$. All the mutants showed similar neutral lipid patterns by TLC analysis, with a small distinct spot exhibiting the same $R_{F}$ value as TAG; this was identified as a residual amount of TAG by preparative TLC and MALDI-TOF, indicating that this bacterium is possibly capable of synthesizing TAGs by at least two different pathways.

Keywords: Rhodococcus, triacylglycerols, storage lipids

\section{INTRODUCTION}

Yeasts and moulds were recognized over a century ago to accumulate lipids (Ratledge, 1992). Since then, other lipid-accumulating micro-organisms have been identified among algae and also a few bacteria (Ratledge, 1989). Lipophilic storage compounds represent a convenient form for storing energy because of their relative

Abbreviations: ELSD, evaporative light-scattering detector; MALDI-TOF, matrix-associated laser desorption ionization-time of flight mass spectrometry; PHA, polyhydroxyalkanoic acid; TAG, triacylglycerol. compactness, being anhydrous, and their much higher calorific value as compared to proteins or carbohydrates (Murphy, 1993). Many bacteria are able to accumulate specialized lipids, such as poly(3-hydroxybutyric acid) or other polyhydroxyalkanoic acids (PHAs) (Steinbüchel, 1991; Steinbüchel \& Valentin, 1995). The accumulation of triacylglycerols (TAGs) has only been described for a few bacteria belonging to the genera Mycobacterium (Barksdale \& Kim, 1977), Streptomyces (Olukoshi \& Packter, 1994; Packter \& Olukoshi, 1995), Acinetobacter (Makula et al., 1975; Scott \& Finnerty, 1976), Nocardia and Rhodococcus (Alvarez et al., 1997). TAGs occur in bacteria as insoluble inclusions sur- 
rounded by a membrane, as reported for Streptomyces coelicolor, S. lividans (Packter \& Olukoshi, 1995) and Rhodococcus opacus PD630 (Alvarez et al., 1996).

Recently, aerobic, Gram-positive bacteria belonging to the genus Rhodococcus have attracted increased interest in the biochemical and genetical characterization of their metabolic capabilities, based on their ability to transform or degrade diverse classes of mainly hydrophobic substances (Finnerty, 1992; Warhust \& Fewson, 1994). Rhodococcus spp. are also able to synthesize and accumulate PHAs after cultivation on different carbon sources under nitrogen-limiting conditions. The accumulation of a copolymer consisting of 3-hydroxybutyrate and 3-hydroxyvalerate from unrelated carbon sources was reported for Rhodococcus ruber (Haywood et al., 1991). Alvarez et al. (1996, 1997) reported on an oleaginous hydrocarbon-degrading $R$. opacus strain, PD630, which is able to accumulate up to $76 \%$ of acylglycerols when grown on different carbohydrate and non-carbohydrate carbon sources under nitrogenlimiting conditions but is unable to synthesize PHAs.

The present work is concerned with the analysis of the composition and stereospecific structures of storage acylglycerols from $R$. opacus PD630, which have not been studied in detail previously. Stereospecific analysis of TAGs has been performed for many years to discriminate between fatty acids at the $s n-1,-3$ and -2 positions of TAG (Brockerhoff, 1965). In addition, we subjected R. opacus PD630 to chemical mutagenesis and screened for mutants defective in the accumulation of storage lipids.

\section{METHODS}

Bacterial strains, media and growth conditions. $R$. opacus PD630 (DSMZ 44193; Alvarez et al., 1996) and the mutant $R$. opacus ROM34 derived from this strain (Kalscheuer et al., 1999) were grown aerobically at $30{ }^{\circ} \mathrm{C}$ in $0.8 \%(\mathrm{w} / \mathrm{v})$ nutrient broth medium or in mineral salts medium (MSM) according to Schlegel et al. (1961) with $1 \%(\mathrm{w} / \mathrm{v})$ sodium gluconate as carbon source. The concentration of ammonium chloride was reduced to $0.01 \%(\mathrm{w} / \mathrm{v})$ to promote the accumulation of TAGs. To obtain solidified media, $1.8 \%(\mathrm{w} / \mathrm{v})$ agar was added.

Lipid extraction and TLC. Crude oil of R. opacus PD630 was obtained from lyophilized cell material ( $15 \mathrm{~g})$, which was stirred twice each with $500 \mathrm{ml}$ chloroform/methanol (2:1, $\mathrm{v} / \mathrm{v}$ ) at room temperature for $3 \mathrm{~h}$. The solvents were evaporated from the combined extracts, and the residual material, $5 \cdot 2 \mathrm{~g}$, was dissolved in light petroleum (b.p. $30-40{ }^{\circ} \mathrm{C}$ ) and applied to a column packed with $20 \mathrm{~g}$ silica gel 60 (mesh 50-200 $\mu \mathrm{m}$, Merck) equilibrated with light petroleum. The column was eluted with $500 \mathrm{ml}$ light petroleum; the total eluate was collected and evaporated to dryness to obtain $4.4 \mathrm{~g}$ of a yellowish crude oil fraction, which consisted of $90 \%$ fatty acids in total.

To determine the identity of the lipids, TLC was carried out on silica gel $60 \mathrm{~F}_{254}$ plates $(0 \cdot 2 \mathrm{~mm}$, Merck). For this, samples of lyophilized cell material were extracted with chloroform/ methanol $(2: 1, \mathrm{v} / \mathrm{v})$, and light petroleum (b.p. $\left.30-40{ }^{\circ} \mathrm{C}\right) /$ diethyl ether/acetic acid (70:30:1) was used as solvent. In some experiments, a double solvent system, benzene/acetic acid $(85: 15, \mathrm{v} / \mathrm{v})$ followed by light petroleum (b.p. $\left.30-40{ }^{\circ} \mathrm{C}\right)$ applied in the same direction, was used. Preparative TLC was carried out on silica gel plates of $2 \mathrm{~mm}$ thickness (Merck). Plates impregnated with methanolic silver nitrate or boric acid (each $5 \%, \mathrm{w} / \mathrm{v}$ ) were also used. Lipid fractions were visualized by heating the plates over a Bunsen flame after spraying with $40 \%$ sulfuric acid. Components on preparative plates were visualized after brief exposure to iodine vapour. Quinone compounds were detected on silica gel TLC plates by specific staining with a leukomethylene blue spray reagent (Krebs et al., 1967). Quinones appeared immediately as dark blue spots on a faint blue background. Osmium tetroxide vapour was used to visualize unsaturated compounds. 2-Monopalmitoylglycerol, tripalmitoylglycerol, 1,2-dipalmitoylglycerol, 1,3dipalmitoylglycerol, cetylpalmitate, 1,2-dipalmitoyl-3-myristoyl-rac-glycerol, hexadecanol, tetradecene and oleic acid obtained from Sigma were used as reference substances.

Analysis of fatty acids. To determine the fatty acid content of the cells and the composition of the lipids, these compounds were treated to yield fatty acid methyl esters (Brandl et al., 1988; Timm et al., 1990), which were analysed by GC with a Perkin Elmer model 8420 gas chromatograph equipped with a Permaphase PEG $25 \mathrm{Mx}$ capillary column $(25 \mathrm{~m} \times 0.32 \mathrm{~mm})$ and a flame ionization detector. A $2 \mu \mathrm{l}$ portion of the organic phase was analysed after split injection (split ratio, 1:40); helium $\left(32 \mathrm{ml} \mathrm{min}^{-1}\right)$ was used as carrier gas. The temperatures of the injector and detector were $230^{\circ} \mathrm{C}$ and $275^{\circ} \mathrm{C}$, respectively, and a temperature programme was used for the separation of methyl esters $\left(120^{\circ} \mathrm{C}\right.$ for $5 \mathrm{~min}$; temperature increase of $8^{\circ} \mathrm{C} \mathrm{min}^{-1} ; 180^{\circ} \mathrm{C}$ for $37 \mathrm{~min}$ ) on the column. The fatty acids were identified by comparison of their retention times with those of standard fatty acid methyl esters.

Reversed-phase HPLC. Reversed-phase HPLC was performed on an RP-18 Merck LiChroSphere 100 column $(5 \mu \mathrm{m}, 250 \times$ $4.6 \mathrm{~mm}$ i.d.) using a Kontron HPLC apparatus equipped with a series 522 chromatographic pump. Elution of the compounds was monitored at $210 \mathrm{~nm}$ using a Kontron DAD 540 diode array detector (DAD) or an Alltech ELSD 500 evaporative light-scattering detector (ELSD) connected to a KromaSystem 2000 integrator system. The ELSD temperature was set to $55^{\circ} \mathrm{C}$. Acetonitrile/acetone $(38: 62, \mathrm{v} / \mathrm{v})$ at a flow rate of $1 \mathrm{ml}$ $\mathrm{min}^{-1}$ was used as solvent system. To characterize TAG mixtures further, a partition number $(P N)$ variable was used: $P N=C N-2 N D$, where $C N$ and ND represent the total carbon number and the total number of double bonds in the fatty acids in the TAG molecule, respectively (Ruiz-Gutiérrez \& Barron, 1995).

Matrix-associated laser desorption ionization-time of flight mass spectrometry (MALDI-TOF). MALDI-TOF was carried out using a LAZARUS III DE time of flight mass spectrometer (constructed by H. Luftmann, Institut für Organische Chemie, Münster, Germany) operated at $19 \mathrm{kV}$ with delayed extraction and a path length of $2 \mathrm{~m}$. A nitrogen laser was used to generate the primary beam, at $337 \mathrm{~nm}$, with $3 \mathrm{~ns}$ pulse width. Samples were applied to the stainless steel target as $10^{-4}$ to $10^{-5}$ solutions $(1 \mu \mathrm{l})$ mixed with an equal volume of a $0.1 \mathrm{M}$ solution of 2,5-dihydroxybenzoic acid. The applied drop was allowed to dry and to crystallize before the sample was introduced into the mass spectrometer ion source.

Stereospecific analysis of TAGs. The procedure for the stereospecific analysis of TAGs is reported in the literature (Brockerhoff, 1965; Yurkowski \& Brockerhoff, 1966; Christie 
\& Moore, 1968) and was applied to R. opacus PD630 crude oil as follows.

(i) Enzymic hydrolysis of TAGs was done with porcine pancreatic lipase (glycerol-ester hydrolase, EC 3.1.1.3; Merck) to obtain $s n$-2-monoacylglycerols.

(ii) Partial deacylation of TAGs was done by chemical hydrolysis with ethyl magnesium bromide (Alfa Aesar).

(iii) Separation of enantiomeric $s n$-1,2(2,3)-diacylglycerols was achieved by TLC on boric-acid-impregnated silica gel plates, followed by treatment with phosphatidyl phenol (Sigma) to obtain synthetic L- and D-phosphatidyl phenols, which were subsequently hydrolysed by stereospecific phospholipase $\mathrm{A}_{2}$ (EC 3.1.1.4) from rattlesnake venom (Crotalus atrox) to obtain the lysophosphatidyl phenols (Brockerhoff, 1965).

(iv) GC analysis of the fatty acid composition of TAGs, sn-2monoacylglycerols and the lysophosphatidyl phenols was done as described above.

Mutagenesis. To obtain mutants deficient in TAG accumulation, R. opacus PD630 was subjected to chemical mutagenesis using $N$-methyl- $N^{\prime}$-nitro- $N$-nitrosoguanidine (NMG). Cells were incubated with $20 \mu \mathrm{g} \mathrm{NMG} \mathrm{ml}{ }^{-1}$ for $30 \mathrm{~min}(50 \%$ lethal rate). For expression of the mutations, the mutagenized cells were washed and resuspended in nitrogen-limited MSM containing $1 \%(\mathrm{w} / \mathrm{v})$ sodium gluconate. Enrichment of mutants was performed by Percoll density-gradient ultracentrifugation (Guerrero et al., 1984). Since the buoyant density of cells of TAG-deficient mutants was higher than that of the wild-type cells, two distinct bands were obtained. The band representing the mutant cells was withdrawn from the gradient, plated on nitrogen-limited MSM agar plates containing $1 \%(\mathrm{w} / \mathrm{v})$ sodium gluconate and identified by staining with ethanolic Sudan Black B and subsequent destaining with ethanol (Schlegel et al., 1970).

\section{RESULTS}

\section{TLC pattern of neutral lipids of $R$. opacus PD630}

The lipids from the crude organic extract were separated by TLC, isolated from the plates, and the structures of neutral storage lipids were elucidated by spectrometric methods. The TAG fraction accounted for about $90 \%$ $(\mathrm{w} / \mathrm{w})$ of the total extractable lipids of $R$. opacus PD630. The spectrometric data obtained were compared with published data and with data from commercially available standards, resulting in the identification of TAG, 1,2-diacylglycerol and 1,3-diacylglycerol (see Fig. 2). A thin-layer chromatogram of the crude organic extract and the determined structures is shown in Fig. 1. The pattern of neutral lipids separated on TLC plates also included some compounds not representing storage lipids because of their relatively low concentration found in the extractable lipids, and some signals originated from the solvents and artefacts of extraction (referred to as 'unknown'). The intensities of these signals corresponded to the solvent volume used for cell extraction or were referred to the solvent systems used for TLC. Quinones were detected by specific staining on TLC plates with leukomethylene blue and are indicated in Fig. 1.

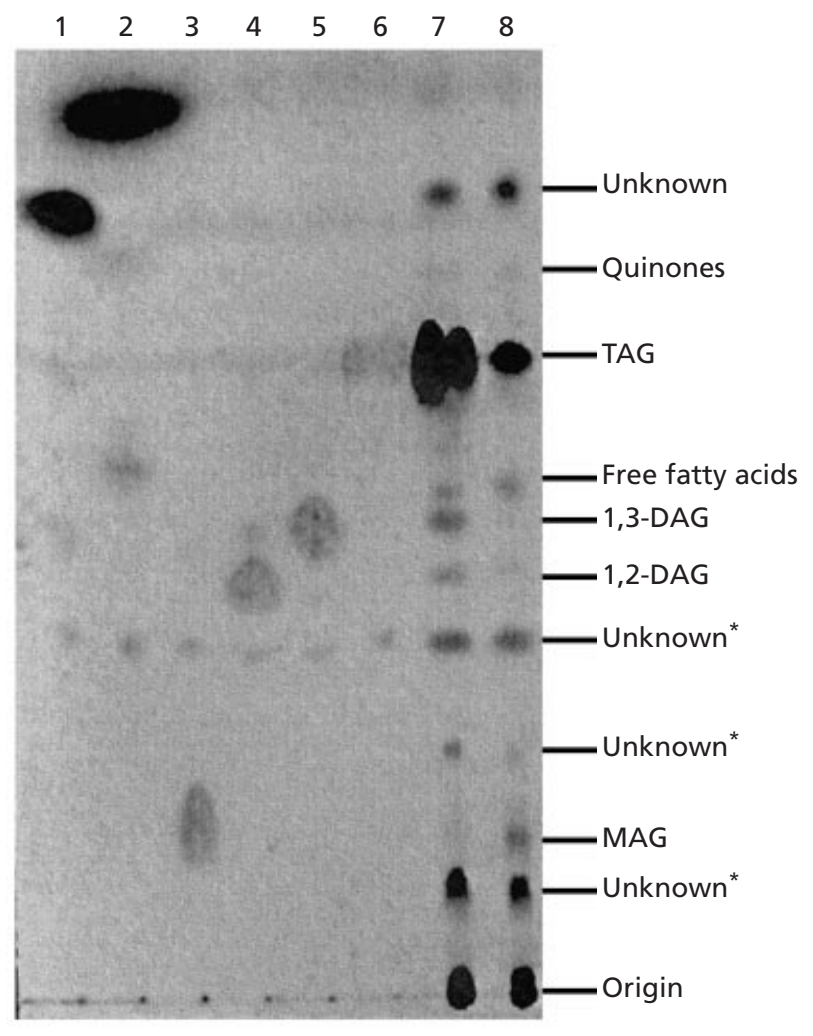

Fig. 1. TLC analysis of the crude organic extracts obtained from $R$. opacus PD630 and the TAG-deficient mutant $R$. opacus ROM70. The cells were grown in nitrogen-limited MSM containing $1 \%(\mathrm{w} / \mathrm{v})$ sodium gluconate for $48 \mathrm{~h}$. Lipids were extracted and separated by TLC on silica gel plates as described in Methods. Solvent system: benzene/acetic acid (85:15, $\mathrm{v} / \mathrm{v}$ ) and then light petroleum (b.p. $30-40^{\circ} \mathrm{C}$ ) in the same direction. Lanes: 1, Cetylpalmitate; 2, tetradecene; 3, 2-monopalmitoylglycerol; 4, 1,2-dipalmitoylglycerol; 5, 1,3dipalmitoylglycerol; 6, tripalmitoylglycerol; 7, crude lipid extract of $R$. opacus PD630; 8 , crude lipid extract of $R$. opacus ROM70. DAG, diacylglycerol; MAG, monoacylglycerol. *Spots may result from impurities in the extraction solvent.

\section{Fatty acid profile of accumulated TAGs of $R$. opacus PD630}

The profile of fatty acids found in TAGs purified from gluconate-grown cells of $R$. opacus PD630 corresponded well with the fatty acid composition obtained from the analysis of intact cells (Table 1) and was in good agreement with those reported previously (Alvarez et al., 1996). The fatty acids ranged in chain length from 13 to 19 carbon atoms, with the major fatty acids being 16:0, 17:0, 17:1 and 18:1. The only monomethylbranched fatty acid that could be detected in the purified TAGs was tuberculostearic acid, present at trace levels $(<0.2 \%, \mathrm{w} / \mathrm{w})$; otherwise no other iso- or anteisofatty acids were detectable, as revealed by GC analysis at the Deutsche Stammsammlung von Mikroorganismen und Zellkulturen (Braunschweig, Germany). Therefore, the odd-numbered fatty acids were almost exclusively straight-chain fatty acids. Dienic fatty acids were not 
Table 1. Distribution of fatty acids in TAGs from $R$. opacus PD630

Cells were cultivated for $48 \mathrm{~h}$ in low-nitrogen medium with $1 \%(\mathrm{w} / \mathrm{v})$ sodium gluconate at $30{ }^{\circ} \mathrm{C}$. Abbrevations and symbols: $\mathrm{ND}$, not detectable; tr, traces $(<0.5 \%) ;-$, could not be calculated.

\begin{tabular}{|c|c|c|c|c|c|c|c|c|c|c|}
\hline & \multicolumn{10}{|c|}{ Fatty acid $(\mathrm{mol} \%)$} \\
\hline & C13:0 & $\mathrm{C} 14: 0$ & C15:0 & C16:0 & C16: 1 & $\mathrm{C} 17: 0$ & $\mathrm{C} 17: 1$ & C18:0 & C18: 1 & $\mathrm{C} 19: 0$ \\
\hline Crude extract & ND & $5 \cdot 2$ & $6 \cdot 3$ & $25 \cdot 9$ & $9 \cdot 5$ & $12 \cdot 6$ & $15 \cdot 4$ & $3 \cdot 1$ & $22 \cdot 0$ & $\mathrm{ND}$ \\
\hline Purified TAG & $0 \cdot 8$ & $4 \cdot 3$ & $6 \cdot 3$ & $25 \cdot 7$ & $9 \cdot 5$ & $12 \cdot 3$ & $15 \cdot 4$ & $3 \cdot 5$ & $22 \cdot 0$ & $\operatorname{tr}$ \\
\hline$s n-2$ & ND & $11 \cdot 3$ & $11 \cdot 7$ & $59 \cdot 3$ & $\operatorname{tr}$ & $17 \cdot 6$ & $\mathrm{ND}$ & $\operatorname{tr}$ & $\operatorname{tr}$ & $\mathrm{ND}$ \\
\hline$s n-1$ & ND & $12 \cdot 0$ & $3 \cdot 4$ & $18 \cdot 4$ & $7 \cdot 1$ & $12 \cdot 0$ & $16 \cdot 2$ & $6 \cdot 0$ & $23 \cdot 7$ & $\mathrm{ND}$ \\
\hline$s n-3$ (calculated) & - & - & $3 \cdot 8$ & $-0 \cdot 6$ & $21 \cdot 4$ & $7 \cdot 3$ & $30 \cdot 0$ & $4 \cdot 5$ & $42 \cdot 3$ & - \\
\hline
\end{tabular}
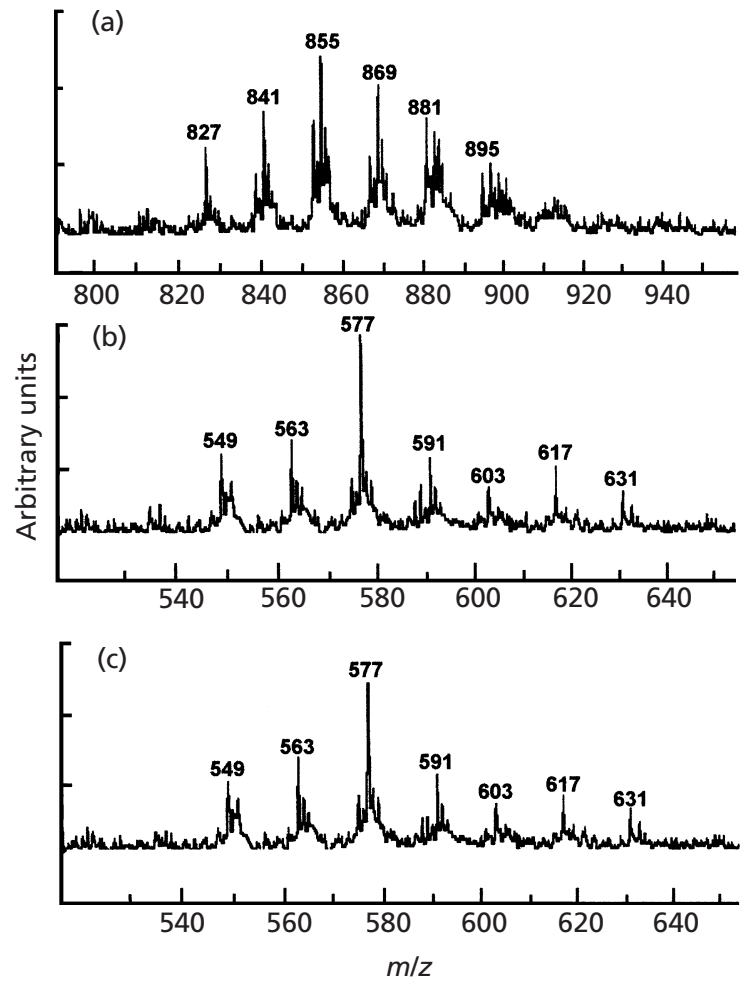

Fig. 2. MALDI-TOF spectra of acylglycerols of $R$. opacus PD630 obtained after preparative TLC. (a) TAG fraction; (b) 1,2diacylglycerol fraction; (c) 1,3-diacylglycerol fraction. All assignments correspond to $[\mathrm{M}+\mathrm{Na}]^{+}$.

detected. The saturated fatty acid content was over $52 \%$, and the content of odd-numbered fatty acids was about $35 \%$. Octadecenoic acid was identified as oleate $\left(\Delta^{9}\right.$-octadecenoic acid) and was the major monoenic fatty acid.

\section{Mass distribution of acylglycerols accumulated by R. opacus PD630}

Following preparative TLC of acylglycerols, MALDITOF mass spectral data were acquired in order to obtain the molecular masses of the acylglycerols. MS data for

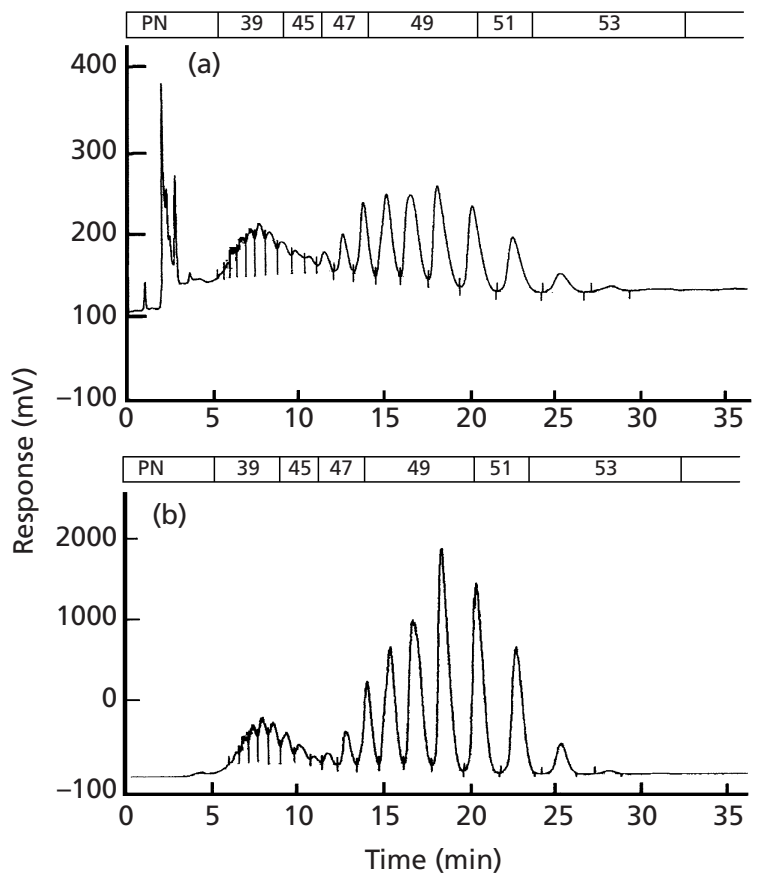

Fig. 3. HPLC separation of purified TAGs of $R$. opacus PD630 using an RP-18 Merck LiChroSphere column $(5 \mu \mathrm{m}, 250 \mathrm{~mm}$ length $\times 4.6 \mathrm{~mm}$ inner diameter). Column temperature, $30^{\circ} \mathrm{C}$; mobile phase, acetonitrile/acetone $(38: 62, \mathrm{v} / \mathrm{v})$ isocratic; flow rate, $1 \mathrm{ml} \mathrm{min}^{-1}$. (a) UV detection at $210 \mathrm{~nm}$; (b) detection by ELSD.

the purified TAG fraction revealed pseudomolecular ions $[\mathrm{M}+\mathrm{Na}]^{+}$corresponding to total carbon units from 51 to 57 (Fig. 2a). MALDI-TOF spectra of purified 1,2- and 1,3-diacylglycerols (Figs $2 \mathrm{~b}$ and 2c, respectively) revealed pseudomolecular ions corresponding to total carbon units from 32 to 38 , indicating a similar fatty acid distribution as in the purified TAGs.

\section{Reversed-phase HPLC}

The reversed-phase HPLC analysis of purified TAG was carried out using UV detection at $210 \mathrm{~nm}$ and an ELSD to detect and distinguish between saturated and un- 
(a)

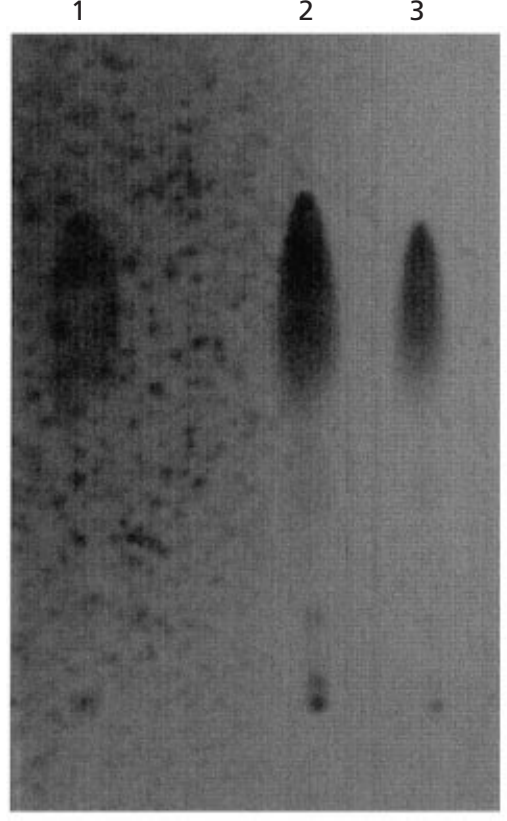

(b)

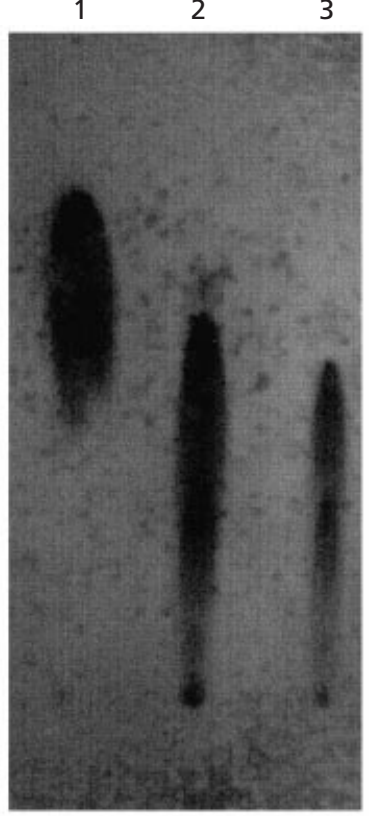

(c)

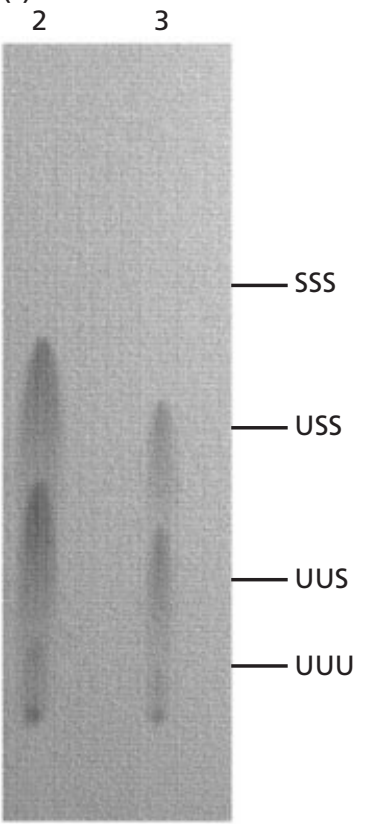

Fig. 4. Separation of TAGs from R. opacus PD630 on a silver-nitrate-impregnated silica gel TLC plate. (a) The TLC plate was not impregnated; $(\mathrm{b}, \mathrm{c})$ the TLC plate was impregnated with $5 \% \mathrm{AgNO}_{3}$. Plates (a) and (b) were stained with $40 \%$ sulfuric acid in the heat, whereas plate (c) was stained with osmium tetroxide vapour to visualize only unsaturated compounds. Lanes: 1, tripalmitoylglycerol $(100 \mu \mathrm{g})$; 2, purified TAGs $(200 \mu \mathrm{g}) ; 3$, purified TAGs $(100 \mu \mathrm{g})$. UUU, TAGs with three monoenic fatty acid residues; UUS, TAGs with two monoenic fatty acid residues; USS, TAGs with one monoenic fatty acid residue; SSS, TAGs with only saturated fatty acid residues.

saturated TAGs. The chromatograms showed approximately 20 individual peaks with estimated $P N$ values between 39 and 53 ; UV and ELSD detection gave similar peak patterns (Figs $3 \mathrm{a}$ and $3 \mathrm{~b}$, respectively). Signals corresponding to $P N$ values between 49 and 51 represented the most abundant peaks.

\section{Argentation TLC}

Purified TAGs of $R$. opacus PD630 were applied to silver-nitrate-impregnated TLC plates. The method of argentation TLC is based on the ability of olefinic bonds to form $\pi$-complexes with silver ions, enabling the separation of substances with different degrees of unsaturation. Under these conditions saturated TAGs migrate virtually with the solvent front, followed by the unsaturated TAGs in the order of an increasing degree of unsaturation. Since R. opacus PD630 accumulates only saturated and monoenic fatty acids, this led to four different spots on the argentation TLC plate, with two abundant spots for TAGs containing one and two monoenic fatty acids (Fig. 4).

\section{Stereospecific analysis of TAGs of $R$. opacus PD630}

The results of the stereospecific analysis are also shown in Table 1; they revealed a rather asymmetric distribution of fatty acids at the glycerol. The shorter and saturated fatty acids tend to prefer position 2 against positions 1 and 3 . The unsaturated fatty acids are predominantly found at position 3. Unsaturated fatty acids and acids with more than 17 carbon atoms were not found in significant amounts at position 2. Only small amounts of saturated fatty acids were found at position 3; palmitic acid does not appear to occur at this position. Therefore, the TAGs from R. opacus PD630 were quite different from those of mammals and plants, where the unsaturated longer fatty acids are predominantly found at position 2 .

\section{Acylglycerol accumulation in mutants of $R$. opacus PD630}

R. opacus PD630 was subjected to chemical mutagenesis using NMG and screened for mutants defective in the accumulation of storage lipids by staining colonies with Sudan Black B. By this method we obtained more than 100 mutants (referred to as ROM, for Rhodococcus opacus mutants) which all exhibited a significantly lower content of TAGs and 1,2-diacylglycerols in comparison with the wild-type. They all also lacked detectable 1,3-diacylglycerols. In contrast, the amounts of monoacylglycerols were slightly higher as revealed by TLC analysis (see ROM70 in Fig. 1 as an example). A recorded MALDI-TOF spectrum with crude lipid extracts of mutant ROM34 did not indicate accumulation 


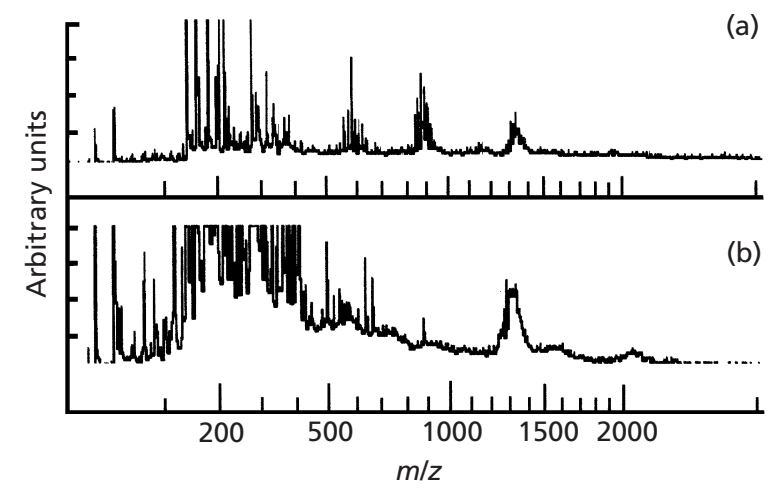

Fig. 5. MALDI-TOF spectra of lipid crude extracts of (a) $R$. opacus PD630 and (b) R. opacus ROM34.

of any TAGs (Fig. 5). To identify the structure of the compound found at the same $R_{\mathrm{F}}$ value as TAGs in the TLC analysis, it was purified by preparative TLC, and the concentrated sample was analysed by MALDI-TOF. This analysis showed that it consisted of a similar mixture of TAGs to the wild-type. For further characterization of the isolated mutants, the spectra of the fatty acids which were incorporated into the various accumulated lipids were also analysed by GC after extraction and methanolysis. All mutants possessed a similar GC profile (data not shown). The amounts of the accumulated fatty acids were drastically reduced in all mutants, to less than $1 \%$ as compared to the wild-type. Therefore, the occurrence of monoacylglycerols in the mutants did not compensate for the lower TAG content. It is therefore obvious that all mutants were still able to synthesize and accumulate trace amounts of TAGs.

\section{Investigation of wax ester accumulation}

TLC analysis of R. opacus PD630 crude extracts (Fig. 1) revealed a faint signal with the same $R_{\mathrm{F}}$ value as cetylpalmitate; this signal was formerly referred to wax ester accumulation (Alvarez et al., 1996). The compound was purified by preparative TLC and subjected to MALDI-TOF and electron-impact mass spectrometry (EI-MS) analysis, but data obtained by these methods did not confirm the presence of any wax ester (data not shown). Specific staining on TLC plates with leukomethylene blue revealed a faint signal for this compound, indicating that it might contain a quinone structure. The recorded MALDI-TOF spectrum of this compound revealed two major signals differing in the length of two isoprene units with $m / z$ values $[\mathrm{M}+\mathrm{Na}]^{+}$ of 550 and 685, which supports the presence of an additional quinone compound, but exact identification of these compounds could not be achieved.

\section{DISCUSSION}

R. opacus PD630 produced significant amounts of various acylglycerols that occurred as insoluble inclusions in the cells when the cells were cultivated on sodium gluconate under nitrogen-limiting conditions. We demonstrated that the major fraction of the fatty acids accumulated by $R$. opacus PD630 are covalently linked to glycerol and occur as TAGs together with traces of diacylglycerols as revealed by TLC, GC and MALDI-TOF analysis. We showed that the pattern of lipophilic lipids seen in TLC analysis also contained some compounds which do not appear to function as storage lipids. Former indications of small amounts of wax esters (Alvarez et al., 1997) could not be confirmed.

Stereospecific analysis of $R$. opacus PD630 TAGs to determine the distribution of fatty acids linked to either position of the glycerol backbone showed that fatty acids in the TAGs of R. opacus PD630 are not randomly distributed between the three possible positions. In contrast to common plant fats, where the longer and higher unsaturated fatty acids are found in position sn2, R. opacus PD630 accumulated only the shorter and saturated fatty acids in this position. This indicates that the enzymes involved in TAG biosynthesis in R. opacus PD630 are highly specific and exhibit a substrate range different from the corresponding enzymes in plants.

In addition, we found that $R$. opacus PD630 synthesizes oleic acid $\left(18: 1^{\Delta 9, c i s}\right)$, whereas vaccenic acid $\left(18: 1^{\Delta 11, c i s}\right)$ was not detected. This provides evidence that $R$. opacus PD630 possesses the aerobic mechanism of fatty acid desaturation (Bloomfield \& Bloch, 1960; Fulco et al., 1964).

All mutants defective in TAG accumulation obtained in this study still accumulated very small amounts of TAG, which were less than approximately $1 \%$ of the wildtype. The results support the two following explanations. The remaining amounts of TAG could be explained by the existence of two different pathways for the biosynthesis of TAGs in R. opacus PD630. One pathway could be only slightly active and contribute only very small amounts of TAG in both the wild-type and the mutants. The second, major, pathway could be responsible for the accumulation of the major fraction of TAGs. If only one pathway existed, completely TAGnegative mutants should have occurred among the more than 100 mutants that were isolated and screened in this study. It is implied that all the mutants isolated were most likely defective in the major pathway; mutants impaired in the minor pathway were probably not detectable by the screening method applied, using the lipophilic dye Sudan Black B, due to the putatively only faint differences in the amounts of TAGs between the wild-type and this type of mutant that were expected. On the other hand, however, it is not excluded that all the mutants isolated were substantially impaired in the regulation of TAG production. This latter possibility could also lower the rate of TAG biosynthesis and give a similar mutant phenotype.

\section{ACKNOWLEDGEMENTS}

This study was supported partly by Monsanto Life Science Company (St Louis, MO, USA) and the Max-Buchner Forschungsstiftung (Frankfurt a. M., Germany). The authors 
are indebted to Dr E. Fehling (Institut für Biochemie und Technologie der Fette, Bundesanstalt für Getreide-, Kartoffelund Fettforschung, Münster, Germany) for performing the reversed-phase-HPLC analysis. They are also grateful to Professor Dr R. Kroppenstedt (DSMZ, Braunschweig, Germany) for the analysis of branched-chain fatty acids.

\section{REFERENCES}

Alvarez, H. M., Mayer, F., Fabritius, D. \& Steinbüchel, A. (1996). Formation of intracytoplastic lipid inclusions by Rhodococcus opacus strain PD630. Arch Microbiol 165, 377-386.

Alvarez, H. M., Kalscheuer, R. \& Steinbüchel, A. (1997). Accumulation of storage lipids in species of Rhodococcus and Nocardia and effect of inhibitors and polyethylene glycol. Fett/Lipid 99, 239-246.

Barksdale, H. \& Kim, K. S. (1977). Mycobacterium. Bacteriol Rev 41, 217-372.

Bloomfield, D. K. \& Bloch, K. (1960). The formation of $\Delta 9$ unsaturated fatty acids. J Biol Chem 235, 337-345.

Brandl, H., Goss, R. A., Lenz, R. W. \& Fuller, R. C. (1988). Pseudomonas oleovorans as a source of poly( $\beta$-hydroxyalkanoates) for potential applications as biodegradable polyesters. Appl Environ Microbiol 54, 1977-1982.

Brockerhoff, H. (1965). Stereospecific analysis of triglycerides: an analysis of human depot fat. Arch Biochem Biophys 110, 586-592.

Christie, W. W. \& Moore, J. H. (1968). A semimicro method for the stereospecific analysis of triglycerides. Biochim Biophys Acta 176, 445-452.

Finnerty, W. R. (1992). The biology and genetics of the genus Rhodococcus. Annu Rev Microbiol 46, 193-218.

Fulco, A. J., Levy, R. \& Bloch, K. (1964). The biosynthesis of $\Delta^{9}$ and $\Delta^{5}$ monounsaturated fatty acids by bacteria. J Biol Chem 239, 998-1003.

Guerrero, R., Mas, J. \& Pedrós-Alió, C. (1984). Buoyant density changes due to intracellular content of sulfur in Chromatium warmingii and Chromatium vinosum. Arch Microbiol 137, 350-356.

Haywood, G., Anderson, A., Williams, D., Dawes, E. \& Ewing, D. (1991). Accumulation of a poly(hydroxyalkanoate) copolymer containing primarily 3-hydroxyvalerate from simple carbohydrate substrates by Rhodococcus ruber NCIMB 40126. Int J Biol Macromol 13, 83-88.

Kalscheuer, R., Arenskötter, M. \& Steinbüchel, A. (1999). Establishment of a gene transfer system for Rhodococcus opacus PD630 based on electroporation and its application for recombinant biosynthesis of poly(3-hydroxyalkanoic acids). Appl Microbiol Biotechnol 52, 508-515.

Krebs, K. G., Heusser, D. \& Wimmer, H. (1967). Sprühreagenzien. In Dünnschichtchromatographie - Ein Laboratoriumshandbuch, pp. 813-861. Edited by E. Stahl. Berlin, Heidelberg \& New York: Springer.
Makula, R. A., Lockwood, P. J. \& Finnerty, W. R. (1975). Comparative analysis of lipids of Acinetobacter species grown on hexadecane. J Bacteriol 121, 250-258.

Murphy, D. J. (1993). Structure, function and biogenesis of storage lipid bodies and oleosins in plants and other organisms. Prog Lipid Res 29, 299-324.

Olukoshi, E. R. \& Packter, N. M. (1994). Importance of stored triacylglycerols in Streptomyces: possible carbon source for antibiotics. Microbiology 140, 931-943.

Packter, N. M. \& Olukoshi, E. R. (1995). Ultrastructural studies of neutral lipid localisation in Streptomyces. Arch Microbiol 164, 420-427.

Ratledge, C. (1989). Biotechnology of oils and fats. In Microbial Lipid, vol 2, pp. 567-650. Edited by C. Ratledge \& S. G. Wilkinson. San Diego: Academic Press.

Ratledge, C. (1992). Microbial lipids: commercial realities or academic curiosities. In Industrial Applications of Single Cell Oils, pp. 1-15. Edited by D. J. Kyle \& C. Ratledge. Champaign, IL: American Oil Chemists' Society.

Ruiz-Guitérrez, V. \& Barron, L. J. R. (1995). Methods for the analysis of triacylglycerols. J Chromatogr B671, 133-168.

Schlegel, H. G., Kaltwasser, H. \& Gottschalk, G. (1961). Ein Submersverfahren zur Kultur wasserstoffoxydierender Bakterien: wachstumsphysiologische Untersuchungen. Arch Mikrobiol 38, 209-222.

Schlegel, H. G., Lafferty, R. \& Kraus, I. (1970). The isolation of mutants not accumulating poly- $\beta$-hydroxybutyric acid. Arch Mikrobiol 71, 283-294.

Scott, C. C. L. \& Finnerty, W. R. (1976). Characterization of intracytoplastic hydrocarbon inclusions from the hydrocarbonoxidizing Acinetobacter species H01-N. J Bacteriol 127, 481-489.

Steinbüchel, A. (1991). Polyhydroxyalkanoic acids. In Biomaterials, pp. 123-213. Edited by D. Byrom. London: Macmillan.

Steinbüchel, A. \& Valentin, H. E. (1995). Diversity of polyhydroxyalkanoic acids. FEMS Microbiol Lett 128, 219-228.

Timm, A., Byrom, D. \& Steinbüchel, A. (1990). Formation of blends of various poly(3-hydroxyalkanoic acids) by a recombinant strain of Pseudomonas oleovorans. Appl Microbiol Biotechnol 33, 296-301.

Warhust, A. M. \& Fewson, C. A. (1994). Biotransformations catalyzed by the genus Rhodococcus. Crit Rev Biotechnol 14, 29-73.

Yurkowski, M. \& Brockerhoff, H. (1966). Fatty acid distribution of triglycerides determined by deacylation with methyl magnesium bromide. Biochim Biophys Acta 125, 55-59.

Received 17 November 1999; revised 1 February 2000; accepted 2 February 2000. 